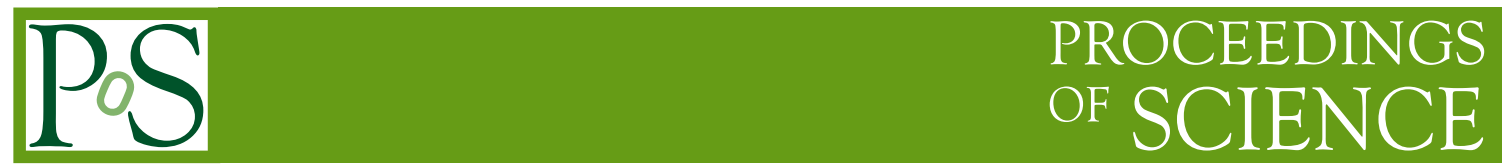

\title{
Double parton scattering results from CMS
}

\author{
Gilvan A. Alves*† \\ CBPF-Centro Brasileiro de Pesquisas Fisicas, Rio de Janeiro, Brazil \\ E-mail: gilvan@cern.ch
}

\begin{abstract}
Double parton scattering (DPS) in proton-proton collisions at a center-of-mass energy of 7 and $8 \mathrm{TeV}$ has been investigated using photon +3 jets, $2 \mathrm{~b}$-jets +2 jets and same-sign $\mathrm{W}$ boson pairs by the CMS detector at the Large Hadron Collider. Data and simulation are used to extract the optimal variables to distinguish double parton scattering from various backgrounds.
\end{abstract}

XXIV International Workshop on Deep-Inelastic Scattering and Related Subjects

11-15 April, 2016

DESY Hamburg, Germany

${ }^{*}$ Speaker.
†On behalf of the CMS collaboration.

(C) Copyright owned by the author(s) under the terms of the Creative Commons 


\section{Introduction}

In high energy proton-proton (pp) collisions at LHC, due to the composite nature of protons, it is possible to have two or more distinct hard parton-parton interactions occurring simultaneously in a single pp collision. At fixed final state invariant masses, such cross sections tend to increase with collision energy because partons with successively lower momentum fractions are being probed. Multiple soft parton-parton collisions are called Multiple parton interactions (MPI), in contrast with those where only a single pair of partons produce a hard scattering, referred as Single Parton Scattering (SPS). Large hadronic activity is observed in the soft regime, characterized by small transverse momenta $\left(p_{T}\right)$ of the produced particles. For relatively large $p T$ values, the observation of MPI will mostly focus on two simultaneous scatterings, i.e. on Double parton scattering (DPS)[1].

The effective cross section, $\sigma_{e f f}$, is a measure of the transverse distribution of partons inside the colliding hadrons and their overlap in a collision. If A and B are two independent processes, whose production cross sections are $\sigma_{A}$ and $\sigma_{B}$, respectively, $\sigma_{\text {eff }}$ can be expressed in terms of the DPS cross section $\left(\sigma_{A+B}^{D P S}\right)$ and the single process cross sections by:

$$
\sigma_{e f f}=\frac{m * \sigma_{A} * \sigma_{B}}{\sigma_{A+B}^{D P S}}
$$

where the symmetry factor $\mathrm{m}=1 / 2$, if processes $\mathrm{A}$ and $\mathrm{B}$ are identical otherwise one.

\section{DPS using same-sign $W$ boson pairs}

A useful feature of same-sign $\mathrm{W}$ boson pair production is the cross section, of the order of $10^{-1} \mathrm{pb}$, comparable for DPS and single parton scattering (SPS), whereas opposite-sign W pair production cross section for SPS events dominates over the DPS one by a factor of $\approx 10^{2}[2]$.

This analysis studied DPS events with two same sign muons and two respectively associated neutrinos in the final state at $8 \mathrm{TeV}[3]$. The data sample corresponds to an integrated luminosity of $19.7 \mathrm{fb}^{-1}$. In case of DPS the two W bosons are produced in first approximation independent of each other, and they are expected to be randomly distributed in the azimuthal plane and with less $p_{T}$ as compared to SPS production. Hence, the muons produced from DPS are less boosted as compared to the leptons produced from SPS and there would not be any correlation between the two muons in the azimuthal plane.

After applying a standard selection for same sign W finals states, background contributions are normalized for the considered integrated luminosity, and simulation-based events are used to evaluate the standard model background of $\mathrm{WZ}, \mathrm{W} \gamma, \mathrm{ZZ}$ and single parton scattering $\mathrm{WW}$ processes. The predicted contribution on the total yield for those processes, after event selection, is less than $30 \%$; the dominant part of background is due to events in which one (or two) muons coming from heavy-flavour decays are misidentified as coming from a prompt decay of $\mathrm{W}$ or $\mathrm{Z}$ boson.

After several control and validation studies, a Multi-Variate Analysis (MVA) using a Boosted Decision Tree (BDT) has been performed in order to improve sensitivity to DPS events with respect to a single observable study. The idea is to use the BDT estimator to get a response shape with the highest possible DPS sensitivity; therefore many sensitive kinematic observables have been put into 
the BDT training process. BDT response is studied on top of the same-sign offline base selection and results are shown in Figure 1.
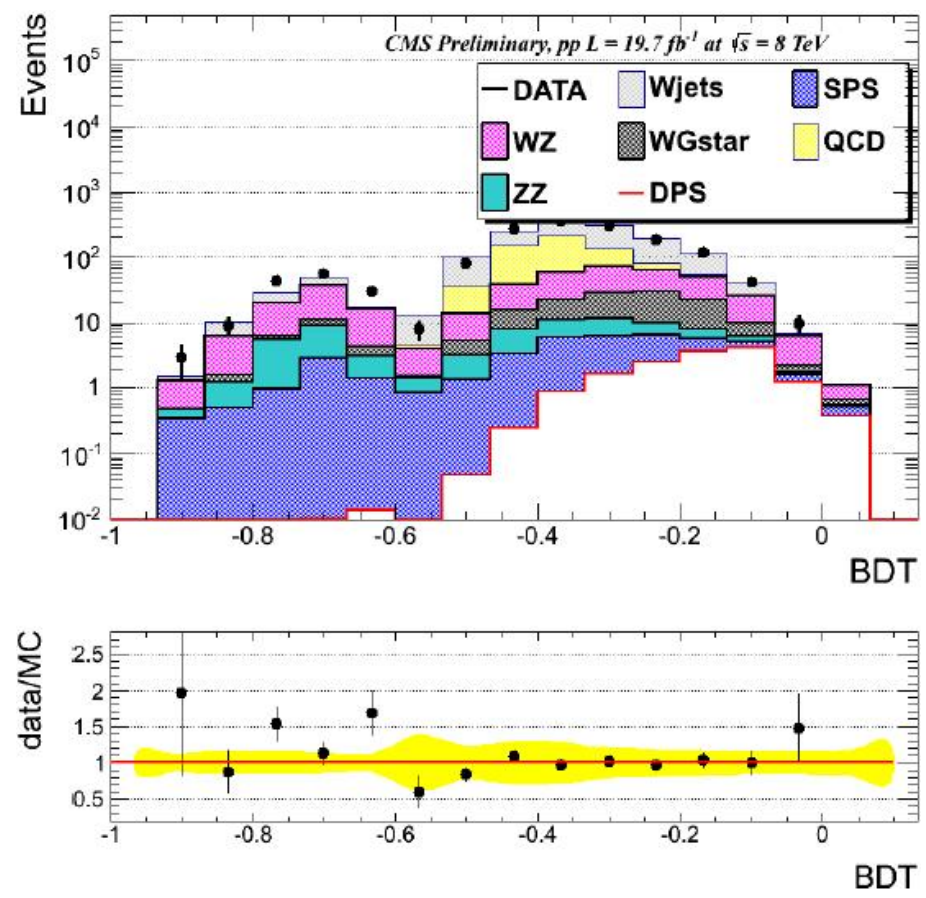

Figure 1: BDT response for events passing offline base selection. Yellow bands are systematics uncertainties, error bars on point are statistical uncertainties.

In order to evaluate a limit on the DPS yield, we computed the expected and observed upper limits on the ratio of measured DPS yield with respect the yield expected from Monte Carlo (signal strength, r) at 95\% Confidence Level (C.L.). We used the CLs method which is based on the modified frequentist approach[4]. Table 1 shows the results for expected and measured limits on DPS yields.

Table 1: 95\% CLs Limit on DPS signal strenght

\begin{tabular}{|l||c|}
\hline \hline 95\% CLs & BDT \\
\hline Expected & $\mathrm{r}<2.001$ \\
Expected $\pm 1 \sigma$ & {$[1.443,2.778]$} \\
Expected $\pm 2 \sigma$ & {$[1.085,3.691]$} \\
\hline Observed & $\mathrm{r}<1.897$ \\
\hline \hline
\end{tabular}

The observed limit can be read as a limit on the DPS same signWboson cross section of:

$$
\sigma_{W W}^{D P S}<r_{\text {observed }} \times \sigma_{W W}^{M C}=1.12 p b
$$

\section{DPS using photon +3 jets}

Distributions sensitive to DPS are investigated in the photon +3 jets final state in pp collisions at $\sqrt{s}=7 \mathrm{TeV}[5]$. The analyzed data sample corresponds to an integrated luminosity of $36 \mathrm{pb}^{-1}$, 
collected by the CMS detector in 2010. The measurement requires the transverse momentum $\mathrm{pT}>$ $75 \mathrm{GeV}$ for the leading (highest $p_{T}$ ) photon and the leading jet and $p_{T}>20 \mathrm{GeV}$ for the other jets. The photon source of DPS signal events can be direct photons and fragmentation photons. Events containing photon +3 jets produced from SPS or misidentified photon $+3 \mathrm{j}$ ets caused by decays of $\pi^{0}$ and $\eta$ mesons constitute an irreducible background. Fig. 2 shows the normalized differential cross section as a function of the azimuthal angle between the $p_{T}$ vectors of the photon-jet pair and the dijet pair defined as:

$$
\Delta S=\Delta \phi\left(\overrightarrow{p_{T}}(\gamma, \text { jet } 1), \overrightarrow{p_{T}}(\text { jet } 2, \text { jet } 3)\right)
$$

where $\gamma$, jet1, jet2, and jet3 stand for the leading photon, the leading jet, the second leading jet, and the third leading jet, respectively. The predictions of PYTHIA 8, MADGRAPH + PYTHIA 8 and SHERPA are compatible with the measured distribution. Switching off the MPI simulation for MADGRAPH + PYTHIA and SHERPA causes about 5-10\% differences. The reason the MPI causes only small changes in $\Delta S$ is due to various background components which reduce the possible sensitivity for the DPS signal contribution.

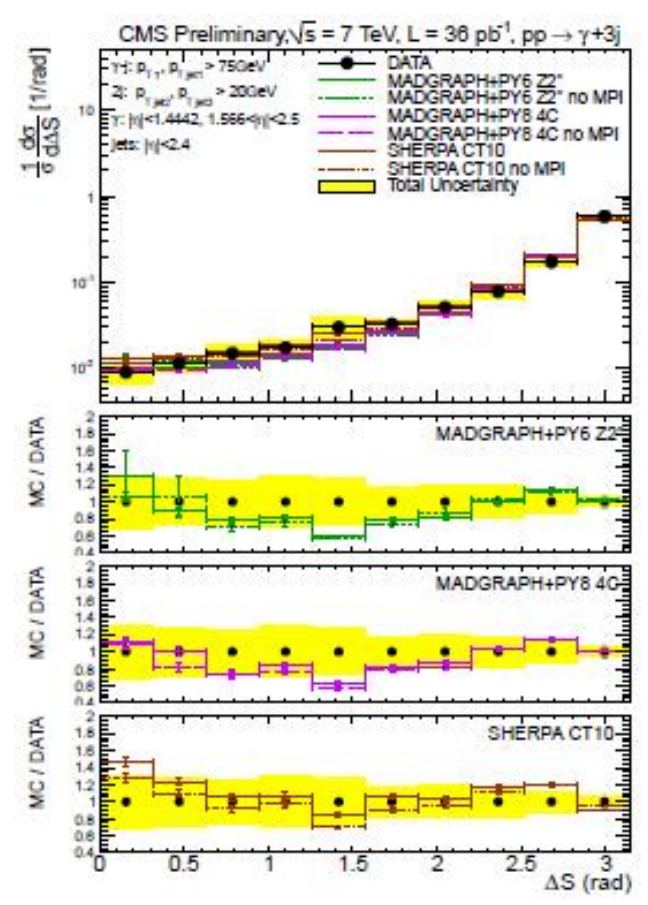

Figure 2: Normalized differential cross sections as a function of $\Delta S$ compared to different MC predictions.

\section{DPS using $2 b$-jets +2 jets}

Measurements of differential cross sections for the production of at least four jets, two of them initiated by a b-quark, in pp collisions have been studied as a function of the transverse momentum $p_{T}$ and pseudorapidity $\eta$, together with the correlations in azimuthal angle and the $p_{T}$-balance among the jets[6]. The data sample was collected in 2010 at $\sqrt{s}=7 \mathrm{TeV}$ with an integrated luminosity of $3 p b^{-1}$. Events with at least four jets with $p_{T}>20 \mathrm{GeV}$ are selected. To study the 
production of pairs of different flavored jets via DPS, the two highest $p_{T}$ jets are associated in the "b-quark jet pair", while the remaining two compose the "light quark jet pair". Fig. 3 shows the differential normalized cross sections as a function of the azimuthal angle, $\Delta S$ between the two dijet pairs (most DPS-sensitive observable), defined as:

$$
\Delta S=\arccos \left(\frac{\overrightarrow{p_{T}}(\text { bottom } 1, \text { bottom } 2) \cdot \overrightarrow{p_{T}}(\text { light } 1, \text { light } 2)}{\mid \overrightarrow{p_{T}}(\text { bottom } 1, \text { bottom } 2)|\cdot| \overrightarrow{p_{T}}(\text { light } 1, \text { light } 2) \mid}\right)
$$

The measured distribution is compared to predictions of POWHEG + PYTHIA 8, MADGRAPH + PYTHIA 8 , PYTHIA 6 , PYTHIA 8 and HERWIG.$++ \Delta S$ is not well described by any prediction: in particular, all of them, except HERWIG ++ and PYTHIA 8 in a lesser extent, underestimate the region at values of $\Delta S<2$. This study shows the need for multiple parton interaction (MPI) contributions in the simulation in order to describe correlation observables between jets.



Figure 3: Normalized cross sections unfolded to the stable particle level as a function of $\Delta S$, compared to different MC predictions.

\section{References}

[1] M. Diehl et al., "Elements of a theory for multiparton interactions in QCD", JHEP 0389 (2012).

[2] J. R. Gaunt, C.-H. Kom, A. Kulesza, and W. J. Stirling, "Same-signWpair production as a probe of double-parton scattering at the LHC", Eur. Phys. J. C 6953 (2010).

[3] The CMS Collaboration, "Double Parton Scattering cross section limit from same-sign W bosons pair production in di-muon final state at LHC", CMS-PAS-FSQ-13-001, (2015).

[4] T. Junk, "Confidence level computation for combining searches with small statistics", Nucl.Instrum.Meth. A434 435Ü443 (1999).

[5] CMS Collaboration, "Study of double parton scattering in photon +3 jets final state in proton-proton collisions at $\sqrt{s}=7 \mathrm{TeV}$, CMSPAS-FSQ-12-017 (2015).

[6] CMS Collaboration, "Studies s of $2 \mathrm{~b}$-jet +2 jet production in proton-proton collisions at $7 \mathrm{TeV}$ ", CMS-PAS-FSQ-13-010 (2015). 